\title{
Toluene-induced leukoencephalopathy with characteristic magnetic resonance imaging findings
}

\author{
Fan Zeng, Heng Yang, Hua-Dong Zhou, Yan-Jiang Wang \\ Department of Neurology and Center for Clinical Neuroscience, Daping Hospital and Institute of Field Surgery, \\ Third Military Medical University, Chongqing 400042, China.
}

\section{A B S T R A C T}

Toluene-induced leukoencephalopathy is a frequently seen medical condition worldwide; however the lack of specific clinical manifestations and laboratory tests makes it difficult to diagnose. Neuroimaging and medical history are often crucial to diagnosis of this disorder. In this report, a case is presented of a patient suffering from toluene-induced leukoencephalopathy with deteriorating cognition impairment and characteristic magnetic resonance imaging (MRI) findings, typified by a "sunflower-like" change in T2-weighted imaging. In addition, the pharmacokinetic properties of toluene are reviewed, as well as the clinical manifestations, typical MRI findings, neuropathology, possible mechanism, and treatment of toluene-induced leukoencephalopathy.

Key words: Leukoencephalopathy, neuroimaging, occupational protection, toluene

\section{INTRODUCTION}

Leukoencephalopathy is a broad term used to describe a number of leukodystrophy-like diseases. Impairment of the nervous system due to toluene inhalation is a frequent source of toxin-induced leukoencephalopathy. Reported symptoms are mostly nonspecific, such as headache, dizziness, and impaired cognition. ${ }^{[1]}$ Toluene-induced leukoencephalopathy may, therefore, go undiagnosed by doctors and neurologists, especially in patients without hematopoietic system impairment. We present here an interesting case of toluene-induced leukoencephalopathy with characteristic magnetic resonance imaging (MRI) findings.

\section{CASE REPORT}

A 44-year-old male patient presented to the clinic with dizziness, headache located primarily in the occipital region, memory decline, and a dull response, occurring

\begin{tabular}{|l|l|}
\hline \multicolumn{2}{|c|}{ Access this article online } \\
\hline Quick Response Code: & \\
\hline & Website: \\
\hline & Www.nnjournal.net \\
& DOI: \\
\hline
\end{tabular}

over a period of 1-month with gradual deterioration. The patient had been a shoemaker for 3 years and had daily contact with toluene-containing glue for around $10 \mathrm{~h}$ a day. The patient had no history of vascular risk factors such as hypertension, diabetes, smoking, or alcohol, and there was no family history of similar symptoms. No other features of note were present in the patient's medical history. Physical and neuropsychological examination revealed memory decline, impaired calculation, and visuospatial impairment, with a mini-mental state examination (MMSE) score of 23. Blood tests showed increased levels of total cholesterol $(6.7 \mathrm{mmol} / \mathrm{L})$ and triglycerides (3.6 mmol/L). Blood cell counts, fasting glucose levels, liver and renal function, and levels of folic acid, vitamin $\mathrm{B}_{12}$, and ferritin were all normal. The possibility of syphilis or human immunodeficiency virus infection was also excluded. Intracranial pressure was $180 \mathrm{mmH}_{2} \mathrm{O}$ as measured by lumbar puncture; however no abnormalities were observed upon further examination of the cerebrospinal fluid. Levels of protein $(0.37 \mathrm{~g} / \mathrm{L})$, glucose levels $(3.67 \mathrm{mmol} / \mathrm{L})$, chloridion (124.4 mmol/L), aspartate transaminase (15.2 U/L), lactate dehydrogenase (15.9 U/L) and adenosine deaminase $(15.18 \mathrm{U} / \mathrm{L})$ were all normal. The total cell count was $0.008 \times 10^{9} / \mathrm{L}$, and the white blood cell count was $0.001 \times 10^{9} / \mathrm{L}$. Pandy's test was negative. No evidence of bacterial infection was

Corresponding Author: Prof. Yan-Jiang Wang, Department of Neurology and Center for Neuroscience, Daping Hospital and Institute of Field Surgery, Third Military Medical University, 10, Changiiang Branch Road, Yuzhong District, Chongqing 400042 , China. E-mail: yanjiang_wang@tmmu.edu.cn 
detected using Gram stain, ink stain, acid-fast stain, or Mycobacterium tuberculosis culture. DNA-based tests for cytomegalovirus, Epstein-Barr virus, and herpes simplex virus were all negative. Chest X-rays, electrocardiogram, and ultrasonography of the heart and abdomen detected nothing unordinary; however, a slight abnormality was observed on the electroencephalogram. Most importantly, cranial MRI of the patient showed diffuse cerebral white matter hyperintensity [Figure 1], which resembled a characteristic "sunflower-like" change in T2-weighted images. The patient was diagnosed with toluene-induced leukoencephalopathy according to the "Diagnostic Criteria of Occupational Acute Toluene Poisoning" of China. After 2 weeks of treatment with mannitol, hyperbaric oxygenation, and neurotrophic medicine including intravenous ganglioside GM1, Vitamins B12, B1, and B6, huperzine-A tablets, and Oxiracetam capsules, the patient's symptoms were mostly resolved, with an MMSE score of 26 on discharge.

\section{DISCUSSION}

As a common component of experimental organic solvents and industrial products such as glues, inks, paints, and paint thinners, toluene is a ubiquitous solvent. The number of people suffering from medical conditions caused by toluene exposure is growing rapidly, particularly among early adolescents experimenting with volatile substances or inhalants and workers in developing countries who lack occupational protection. After inhalation, toluene is quickly absorbed by the lungs, then enters the brain due to its high lipophilicity and accumulates. Following metabolization in the liver, it is excreted by the kidneys mostly in the form of hippuric acid. ${ }^{[2]}$ Chronic toluene inhalation primarily damages the central nervous system (CNS), especially the white matter, and causes toluene-induced leukoencephalopathy and psychosis. ${ }^{[2-4]}$ Although cognitive impairment is the most prominent symptom, additional clinical manifestations may differ depending on the brain regions that are affected, including ataxia, tremors, psychiatric disorders, Parkinson's disease, and temporal lobe epilepsy. ${ }^{[5]}$ The diagnosis of toluene-induced leukoencephalopathy is primarily based upon the history of contact with toluene, clinical manifestations caused by damage to the nervous system, and exclusion of other medical conditions with similar symptoms. Neuroimaging and medical history are crucial to the diagnosis of toluene-induced leukoencephalopathy, as opposed to other types of encephalopathy such as heroin-related encephalopathy or hypoxic-ischemic encephalopathy.

Several studies have focused on the characteristic features of toluene-induced leukoencephalopathy
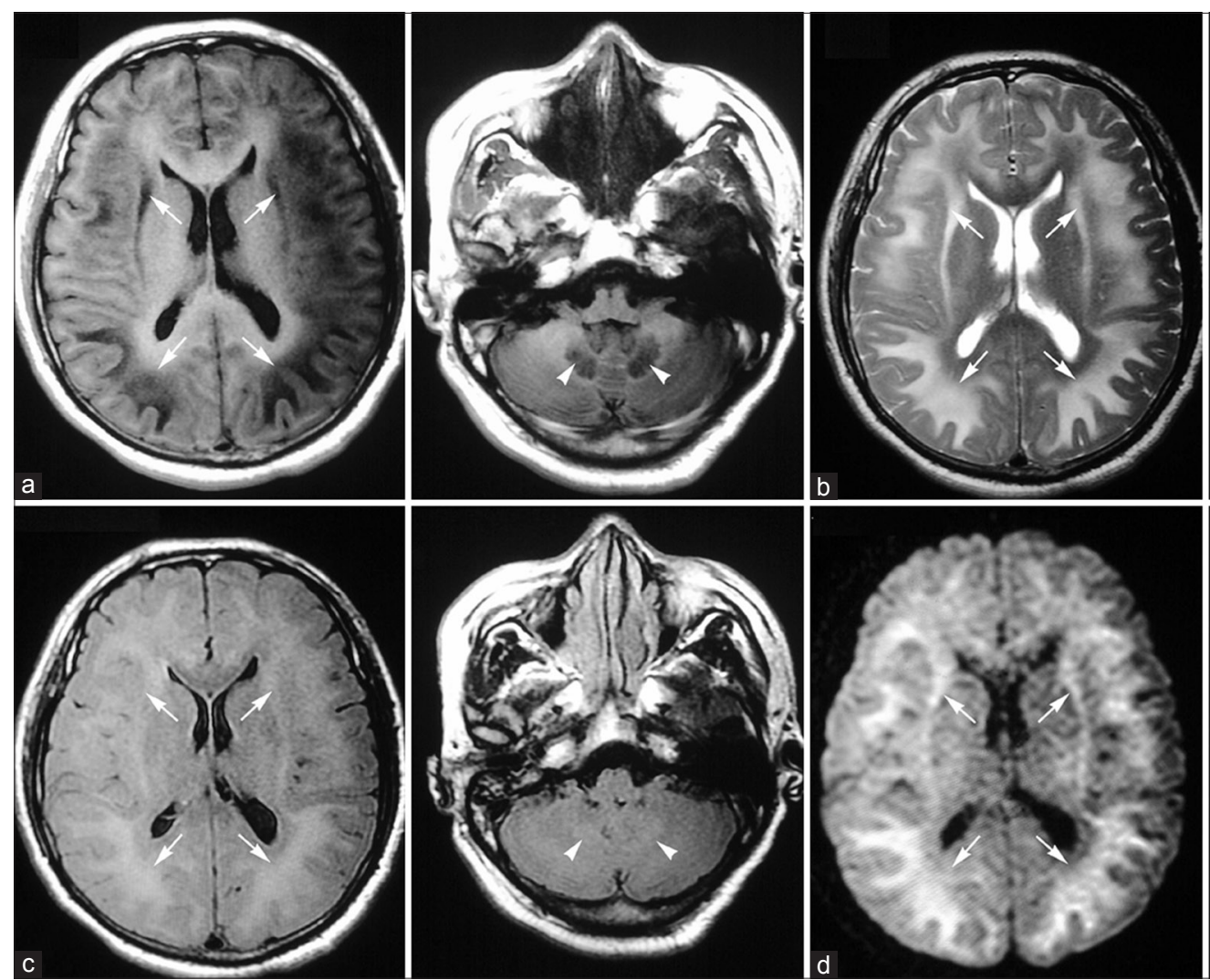
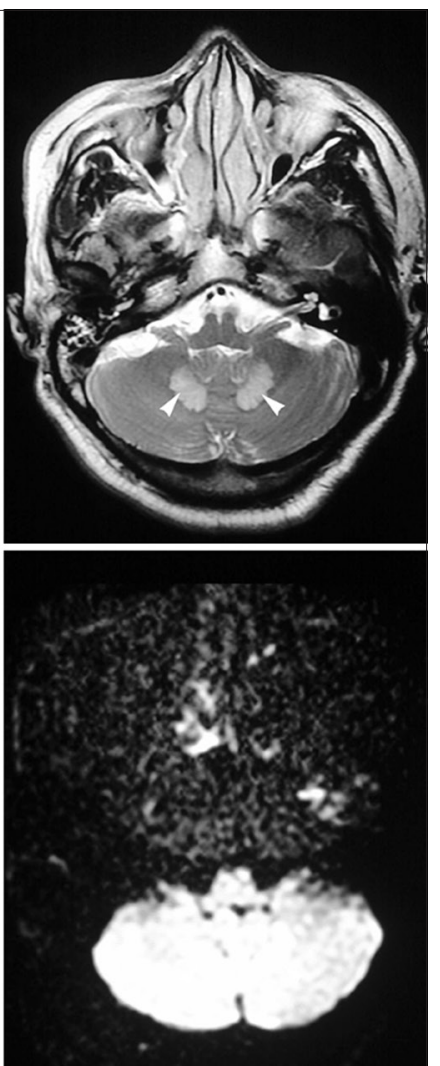

Figure 1: Magnetic resonance imaging shows symmetrical periventricular white matter hyperintensity (arrows) in axial T2-weighted (b), fluid-attenuated inversion recovery (c), and diffusion-weighted imaging (d) images, along with corresponding changes in T1-weighted images (a). The characteristic "sunflower-like" change is readily apparent, particularly in the T2-weighted image. Increased signal is also visible in the cerebellar dentate nuclei (arrowheads). Gray matter-white matter differentiation is preserved 
detectable by neuroimaging. It has been reported that in chronic toluene abusers, MRI often reveals diffuse atrophy of the cerebrum, cerebellum, and brainstem (also discernible with computed tomography), increased periventricular white matter signal on T2-weighted images, and loss of gray matter-white matter differentiation across the cortex. The severity of these abnormalities is considered to be associated with the duration of abuse and concentration of toluene. ${ }^{[2,5]}$ In this case, MRI shows a characteristic "sunflower-like" change and abnormal signal in the cerebellar dentate nuclei [Figure 1], which can be explained by the lipid-dependent distribution of toluene within the CNS and its resulting neuropathology. ${ }^{[1,2,6]}$ Unfortunately, follow-up cranial images were not available for this patient, but according to a previous study, no significant improvement was found in the cranial MRI of a chronic toluene abuser following 5 months of abstinence. ${ }^{[7]}$

Previous neuropathological studies have reported that the brains of chronic toluene abusers show thinning of the corpus callosum and prominent pathology in the periventricular regions. Microscopically, diffuse and ill-defined myelin pallor is seen in cerebral and cerebellar white matter, along with evidence of neuronal loss in the cerebral cortex, basal ganglia, and cerebellum, intense, reactive gliosis, and giant axonal degeneration in the long tracts of the spinal cord..$^{[1,2,6]}$ The degree of myelin and axonal loss is considered to be associated with the extent of exposure, age of onset of toluene abuse, concurrent abuse of other substances, and polymorphisms in the gene encoding the enzyme aldehyde dehydrogenase, which is the main hepatic enzyme responsible for metabolizing toluene.

Although not fully understood, impairment of NMDA, GABA receptor signaling, and mitochondrial function may be involved in the mechanism of toluene-induced leukoencephalopathy. ${ }^{[8,9]}$ Damage to white matter caused by toluene appears to be irreversible, and no treatment is currently known other than abstinence or general neurotrophic compounds. Melatonin was recently reported to reverse the reduction in dendritic branching in animals exposed to toluene, which might be a promising treatment for toluene-induced leukoencephalopathy. ${ }^{[10]}$ Further studies are needed in order to develop more effective therapeutic strategies for this disease.

\section{ACKNOWLEDGMENTS}

This study was supported by Army Health Care Project (13BJZ31).

\section{REFERENCES}

1. Yücel M, Takagi M, Walterfang M, Lubman DI. Toluene misuse and long-term harms: a systematic review of the neuropsychological and neuroimaging literature. Neurosci Biobehav Rev 2008;32:910-26.

2. Filley CM, Halliday W, Kleinschmidt-DeMasters BK. The effects of toluene on the central nervous system. J Neuropathol Exp Neurol 2004;63:1-12.

3. Eller N, Netterstrøm B, Laursen P. Risk of chronic effects on the central nervous system at low toluene exposure. Occup Med (Lond) 1999:49:389-95.

4. Lin BF, Ou MC, Chung SS, Pang CY, Chen HH. Adolescent toluene exposure produces enduring social and cognitive deficits in mice: an animal model of solvent-induced psychosis. World J Biol Psychiatry 2010;11:792-802.

5. Aydin K, Sencer S, Demir T, Ogel K, Tunaci A, Minareci O. Cranial MR findings in chronic toluene abuse by inhalation. AJNR Am J Neuroradiol 2002;23:1173-9.

6. Grandjean P, Landrigan PJ. Developmental neurotoxicity of industrial chemicals. Lancet 2006;368:2167-78.

7. Deleu D, Hanssens Y. Toluene induced postural tremor. J Neurol Neurosurg Psychiatry 2000;68:118.

8. Ahmed S, Tin-Tin-Win-Shwe, Yamamoto S, Tsukahara S, Kunuqita N, Arashidani K, Fujimaki H. Increased hippocampal mRNA expression of neuronal synaptic plasticity related genes in mice chronically exposed to toluene at a low-level human occupational-exposure. Neurotoxicology 2007;28:168-74.

9. Hester SD, Johnstone AF, Boyes WK, Bushnell PJ, Shafer TJ. Acute toluene exposure alters expression of genes in the central nervous system associated with synaptic structure and function. Neurotoxicol Teratol 2011;33:521-9.

10. Pascual R, Bustamante C. Structural neuroplasticity induced by melatonin in entorhinal neurons of rats exposed to toluene inhalation. Acta Neurobiol Exp (Wars) 2011;71:541-7.

Cite this article as: Zeng F, Yang H, Zhou HD, Wang YJ. Toluene-induced leukoencephalopathy with characteristic magnetic resonance imaging findings. Neuroimmunol Neuroinflammation 2014;1(2):92-4

Source of Support: Nil. Conflict of Interest: No.

Received: 27-04-2014; Accepted: 18-07-2014 